\title{
Serum Lipid Levels and Suicide Attempts Within 2 Weeks in Patients With Major Depressive Disorder: Is There a Relationship?
}

\author{
Siyao Zhou ${ }^{1 \dagger}$, Ke Zhao ${ }^{1 \dagger}$, Xiang Shi ${ }^{1}$, Huankun Sun ${ }^{1}$, Siyu Du ${ }^{1}$, Xuemeng Miao ${ }^{1}$, \\ Jianjun Chen ${ }^{1}$, Fan Yang ${ }^{1}$, Minzhi Xing ${ }^{1}$, Wang Ran ${ }^{1}$, Jiaying Lao ${ }^{1}$, Xiangyang Zhang ${ }^{2,3}$, \\ Wei Wang ${ }^{1 *}$ and Wei Tang ${ }^{4 *}$
}

${ }^{1}$ School of Mental Health, Wenzhou Medical University, Wenzhou, China, ${ }^{2}$ Chinese Academy of Science Key Laboratory of Mental Health, Institute of Psychology, Chinese Academy of Sciences, Beijing, China, ${ }^{3}$ Department of Psychology, University of Chinese Academy of Sciences, Beijing, China, ${ }^{4}$ The Affiliated Kangning Hospital of Wenzhou Medical University, Wenzhou, China

\section{OPEN ACCESS}

Edited by:

Yuka Kotozaki,

Iwate Medical University, Japan

Reviewed by:

Domenico De Berardis,

Azienda Usl Teramo, Italy

Siddharth Sarkar

All India Institute of Medical

Sciences, India

*Correspondence:

Wei Tang

tangweikn@163.com

Wei Wang

wangwei@wmu.edu.cn

†These authors have contributed equally to this work

Specialty section:

This article was submitted to

Public Mental Health,

a section of the journal

Frontiers in Psychiatry

Received: 04 March 2021

Accepted: 19 April 2021

Published: 07 June 2021

Citation:

Zhou S, Zhao K, Shi X, Sun H, Du S,

Miao $X$, Chen J, Yang $F$, Xing $M$,

Ran W, Lao J, Zhang X, Wang $W$ and

Tang W (2021) Serum Lipid Levels

and Suicide Attempts Within 2 Weeks

in Patients With Major Depressive

Disorder: Is There a Relationship?

Front. Psychiatry 12:676040.

doi: 10.3389/fpsyt.2021.676040
The potential correlation between serum lipid profiles and suicidal tendencies has been previously reported, however, it is unclear whether serum lipid profiles have definite relevance to recently attempted suicides in individuals suffering from major depressive disorder (MDD). In this study, the relationship between blood lipids and suicide attempts in first-episode MDD patients in research were used to examine whether there is a connection. The cross-sectional study recruited 580 patients at the time of their first episode, measuring up to the diagnostic standard of MDD. Baseline demographic, clinical data, and blood lipid level data were collected. Depression severity was measured with the Hamilton Depression Rating Scale (HAMD). Our results revealed that the level of TC may be identified as a promising and effective biomarker for first-episode MDD suicide risk, suggesting that screening of serum lipid profiles in depressive patients is essential for suicide prevention.

Keywords: depression, suicide unattempted, serum lipid profiles, psychotic, indicator

\section{INTRODUCTION}

As a common psychiatric issue, major depressive disorder (MDD) manifests itself through the characteristics of diminished interest in enjoyable activities, states of low moods, pessimism, cognitive and sleeping disorders, and suicidal behavior $(1,2)$. According to the 2017 report released by the World Health Organization, depression is prevalent in about $4.4 \%$ of the population, with roughly 322 million sufferers worldwide (3). Additionally, roughly 800,000 people die annually through suicide, which forms the second main cause of death in persons aged between 15 and 29 (4). Most cases of suicide ( $\sim 60 \%)$ occur in connection with a background of depressive dysfunction (5), generating a worrisome burden and grave outcome for friends, family, and society. Suicide is a highly complex and multifaceted phenomenon, which may involve multiple mechanisms, such as environmental, familiar, and neurobiological factors as well as temperamental and emotional issues, especially in MDD subjects (6-8). Despite this, research aimed at elucidating the biological indicators related to suicidal behavior, which can serve as an important identifier for prevention and therapeutic action, and remain markedly underdeveloped and understudied (9-11). Research on the underlying causes of suicide that might be related to depressive disorders is strongly warranted. 
Since lipid profiles were demonstrated to form a relationship with depression, considerable research has been conducted in this area over the past few decades. Multiple studies have shown that serum concentration in lipids, primarily measured by levels of total cholesterol (TC), low-density lipoprotein cholesterol (LDL-c), very-low-density lipoprotein cholesterol (VLDL-c), high-density protein cholesterol (HDL-c), and triglycerides (TG) (12), demonstrate correlations with severe depressive disorders. Generally, pro-atherogenic factors are found to incorporate LDLc, VLDL-c, and TG, while anti-atherogenic factors contain HDLc (13). There is some evidence that insufficient levels of HDL-c concentration, ascending ratios of TC/ HDL-c, and abnormal distribution of altered haptoglobin phenotypes are involved in patients with MDD (14-16). Randomized, controlled, and analytic trials have identified that physical training to mediate the lipid spectrum pathway could be used to alleviate depressive symptoms (17). Of note, patients that suffer major depressive episodes also demonstrate a declining and/or low level of cholesteryl esters and an underlying dysfunction of retrograde cholesterol transfer (14).

In the early 1990s, there was some debate over whether serum lipid levels are associated with recent or remote suicide attempts among individuals $(18,19)$. The results of cross-sectional studies concluded that serum cholesterol and triglycerides were not descending in those who had attempted suicide and that excessive levels of cholesterol were, in fact, a risk for suicidal behavior (20-22). Maes et al. (14) identified a positive correlation between low levels of serum HDL-c with depressed individuals who had attempted suicide. A comparison of suicidal subjects and nonsuicidal controls demonstrated that younger and middle-aged adults with suicidal intentions had a higher level of serum TG (23).

Notwithstanding these studies, the research linking lipid profiles with an increased risk of suicide remains inconsistent and conflictual. It is important to note that lowering cholesterol decreases the prevalence of myocardial infarction, which has been clarified by meta-analysis clinical trials, whereas the onset of mortality which is irrelevant to sickness comprising suicidality, force, and occurrence rendered an apparent rise $(24,25)$. Subsequent studies have replicated and confirmed this correlation and it was assumed that suicidal subjects had a reduction of cholesterol concentration $(12,23,26)$, serving as an underlying biological indicator for suicidal tendencies $(27,28)$. However, other studies were unable to discover such a bond, either positive or negative, indicating that the serum cholesterol concentration LDL-c and HDL-c in psychiatric patients who had recently attempted suicide was roughly equivalent to those who had no history of attempted suicide $(11,29)$. These discrepancies have sparked controversial debate and draw attention to the limitations in measuring lipid profiles: namely, a focus on TC concentrations as the dominating measure of lipid profiles while other lipoprotein subfractions are partially omitted (12). Taking into account the heterogeneity of study samples available from around the world, it is difficult to address and replicate the relationship between blood lipid levels and suicide due to a lack of sufficient data to examine dissimilarities between countries $(20,23)$. Additionally, the severity and duration of disorders associated with suicide attempts further complicate results (30).

To date, a range of studies that associate concentrations of serum lipid and suicidality in MDD patients relate to the use of anti-depressants (31), and have tended to ignore the underlying interplay of anti-depressant drugs on the examination of lipid profiles. Moreover, there has been little analysis on the careful measurement of variables concerned with psychopathology and few large sample capacity have been presented in previous studies $(26,32,33)$. In a bid to redress these issues, our study recruited patients diagnosed as first-episode MDD patients who did not take anti-depressants to control the confounding variables and lessen the complications associated with chronic depression and its influence on suicide (34). Patients were selected according to age as this has a significant effect on comparing blood lipids: the level of blood lipids is shown to be positively correlated with age (35). Lastly, patients exhibiting suicidal tendencies within the past 2 weeks were included in this study along with nonsuicidal MDD patients to integrate study variables and observe the fluctuation of lipid concentration.

This study focuses on patients diagnosed with first-episode MDD sufferers, including patients who had recently attempted suicide, to further disentangle the link between lipid profile concentrations and suicidal behavior. We postulate that lipid profiles can be used as a risk indicator of suicidal tendencies among MDD patients.

\section{METHODS}

\section{Subjects}

From July 2011 to October 2017 this cross-sectional study consecutively recruited 662 first-episode patients from a psychiatric outpatient clinic of a hospital who fulfilled the MDD diagnostic criteria confirmed by a psychiatrist. The patients were interviewed by using Structured Clinical Interview for DSM-IV (SCID) to diagnose depression and exclude personality disorder. According to the inclusion and exclusion criteria, we finally include 580 patients in our study, and the specific numbers and conditions of exclusions are as follows: had other neurological or mental disorders $(n=18)$; drug or alcohol abusers $(n=23)$; were pregnant or currently breastfeeding $(n=12)$; had suicide attempts, which did not take place within 2 weeks $(n=29)$. Of all patients, 206 had recently attempted suicide (i.e., within the previous 2 weeks). Before recruitment, we informed all patients of the purpose of the study and all patients had to acknowledge and sign their informed consent before being approved to participate in the study. Ethics approval was obtained from the Affiliated Kangning Hospital of Wenzhou Medical University research ethics committee.

The inclusion criteria required that patients: (1) were of Han nationality; (2) were aged between 18 and 60 years; (3) met the MDD criteria according to the DSM-4; (4) were firstepisode sufferers with no previous history of treatment; (5) achieved a Hamilton Depression Scale-17 (HAMD-17) score of $\geq 24$; and, (6) had recently (i.e., within the previous 2 weeks) attempted suicide. 
TABLE 1 | Socio-demographics, clinical characteristics, and blood lipid profiles between patients with suicide attempts and without suicide attempts.

\begin{tabular}{|c|c|c|c|c|c|c|c|}
\hline \multirow{2}{*}{ Cultural degree } & \multicolumn{2}{|c|}{ MDD with SA ( $n=206)$} & \multicolumn{2}{|c|}{ MDD without SA $(n=374)$} & \multirow{2}{*}{$\begin{array}{l}\mathbf{x}^{2} / \mathbf{t} \\
1.500\end{array}$} & \multirow{2}{*}{$\begin{array}{c}\boldsymbol{P} \\
0.682\end{array}$} & \multirow[t]{2}{*}{ Cohen's d } \\
\hline & & & & & & & \\
\hline Junior high school education level, $n$ (\%) & 58 & 28.2 & 96 & 25.7 & & & \\
\hline Senior high school education level, $n(\%)$ & 85 & 41.3 & 155 & 41.4 & & & \\
\hline University education level, $n$ (\%) & 49 & 23.8 & 103 & 27.5 & & & \\
\hline Post-graduate education level, $n$ (\%) & 14 & 6.7 & 20 & 5.4 & & & \\
\hline Gender & & & & & 1.862 & 0.172 & \\
\hline Male, $n(\%)$ & 57 & 27.7 & 124 & 33.2 & & & \\
\hline Female, $n(\%)$ & 149 & 72.3 & 250 & 66.8 & & & \\
\hline Age, y & 35.62 & 12.49 & 36.65 & 12.60 & 0.951 & 0.342 & $<0.1$ \\
\hline Duration of illness, y & 5.91 & 4.28 & 6.31 & 4.94 & 0.981 & 0.327 & $<0.1$ \\
\hline BMl, kg/m² & 24.67 & 1.76 & 24.45 & 1.83 & -1.436 & 0.152 & 0.12 \\
\hline HAMD & 32.21 & 2.78 & 29.69 & 2.74 & -10.571 & $<0.001^{\star \star \star}$ & 0.91 \\
\hline TC, mmol/L & 5.86 & 1.12 & 5.11 & 1.09 & -7.769 & $<0.001^{\star \star \star}$ & 0.68 \\
\hline $\mathrm{TG}, \mathrm{mmol} / \mathrm{L}$ & 2.29 & 1.04 & 2.12 & 0.98 & -1.850 & 0.065 & 0.17 \\
\hline HDL-c, mmol/L & 1.13 & 0.28 & 1.25 & 0.29 & 4.966 & $<0.001^{\star \star \star}$ & 0.42 \\
\hline LDL-c, mmol/L & 3.20 & 0.92 & 2.93 & 0.87 & -3.504 & $<0.001^{\star \star \star}$ & 0.30 \\
\hline
\end{tabular}

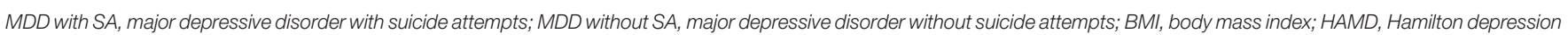
rating scale; TC, total cholesterol; TG, triglycerides; HDL-c, high-density lipoprotein cholesterol; $L D L-c$, low-density lipoprotein cholesterol. ${ }^{\star \star \star} p<0.001$.

The exclusion criteria required that patients: (1) had no other neurological or mental disorders; (2) had not been diagnosed with severe physical illness, such as heart disease, diabetes, organic brain disease, or liver or kidney disease; (3) were not pregnant or currently breastfeeding; (4) were not drug or alcohol abusers or formerly held dependencies on drugs or alcohol; and/or, (5) were not sufferers of hyperlipidaemia.

\section{Clinical Measures}

Prior to the study, eligible psychiatrists were trained for interviews. The study applied HAMD-17 for evaluating the existence and severity of depressive symptoms (36). There are 17 questions on this scale, with a score of $0-4$ points for each item. The higher the score, the more severe the symptoms. In this study, patients with severe depressive symptoms apply at a cut-off point of 24 (37).

The study defines "suicide attempts" as acts of self-harm committed with the conscious intention of ending one's life, but without success (38). A screening question was asked to measure a patient's suicide attempts: "Have you attempted suicide in the last 2 weeks?" If they answered in the affirmative, they were regarded as having recently attempted suicide. If accurate or appropriate answers could not be obtained from the subjects, it was acquired through family, relatives, or friends.

\section{Blood Sample}

Fasting serum samples were taken from 6:00 to 8:00 a.m. after a night of fasting. Blood samples from the subjects, including TG, TC, LDL-C, and HDL-C were used to obtain information about lipid levels. The blood samples of all patients were immediately sent to the hospital testing center before 11:00 to be measured by a chemiluminescence immunoassay using Cobas E610 (Roche, Basel, Switzerland). The normal range for each index was that:
TG (0.56-1.69 mmol/L), TC $(<5.17 \mathrm{mmol} / \mathrm{L})$, LDL-C $(<3.10$ $\mathrm{mmol} / \mathrm{L})$, and HDL-C (1.04-2.07 $\mathrm{mmol} / \mathrm{L})$.

\section{Statistical Analysis}

The data analysis was performed using IBM SPSS Statistics version 19.0. All continuous variables were described by the mean and standard deviation (SD). According to the distribution type, continuous variables were tested by a group $t$-test or a two-sample Mann-Whitney $U$ test. The categorical variables were described by counts and percentages, and the intergroup comparison was conducted using a chi-square test. Moreover, logistic regression analyses were conducted on the entire sample to examine the association between serum lipid profiles and suicide in patients with first-episode MDD, and on age stratification [including gender, age, and body mass index (BMI) as covariables]. Furthermore, a correlation analysis was conducted between HAMD and lipid profiles. A significance level of 0.05 for two-tailed tests was used in the statistical analysis.

\section{RESULTS}

The study population consisted of 580 patients with first-episode MDD (Table 1), divided into a suicide attempt group $(n=206)$ and a non-suicide attempt group $(n=374)$. The HAMD scores, TC, and LDL-c levels were higher for the suicide attempt group $(p<0.05$ or $p<0.001)$ in comparison to the non-suicide attempt group, while the HDL-c levels were lower $(p<0.001)$. There were no statistical differences in age, gender, BMI, duration of illness, culture degree, and the level of TG between the two groups.

Patients were separated into three age cohorts: 18-30 years old ( $n=224), 31-45$ years old $(n=186)$, and $46-60$ years old $(n=170)$. After grouping by age, the general demographic data and blood lipid spectrum of patients were tallied (see 
Table 2). The main results were as follows: In the $31-45$ age cohort, a significantly higher proportion of women than men had attempted suicide $(p=0.027)$. For each age cohort, the suicide attempt group had increased TC levels and HAMD scores $(p<$ 0.001 ). There were no differences recorded in TG levels between the two groups in all three age cohorts, and HDL-c levels were significant $(p<0.05)$, except for in the $46-60$ cohort. The levels of LDL-c were significant $(p<0.05)$, except for in the 31-45 cohort.

Adjusting for age, gender and BMI, logistic regression models evaluated the correlation between recent suicide attempts in MDD patients and the lipid profile for levels of TC $(p<0.001 ; O R=1.897)$ and HDL-c $(p<0.01 ; O R=0.361)$ (Table 3). The association between blood lipids and suicide in the different age cohorts is presented in Table 3. Finally, suicide attempts in MDD patients and TC levels are the only variations in the distinct age cohorts, demonstrating a significant relationship $(p<0.001$ or $p<0.01)$.

The correlation analysis (Table 4) showed that HAMD is correlated with TC $(r=0.584, p<0.001)$, TG $(r=0.209$, $p<0.001)$, HDL-C $(r=-0.200, p<0.001)$, and LDL-C $(r=0.386, p<0.001)$.

\section{DISCUSSION}

This study, by analyzing the lipid profiles of 580 firstepisode MDD patients and other factors, found that suicide attempts were associated with higher scores of HAMD, increased TC, TG, and LDL-c levels of lipid, and decreased HDL-c level were demonstrated in 580 first-episode MDD patients (all $p<0.05$ ). After controlling for other variables and age stratification, the OR of TC was still significant for first-episode MDD patients of all ages. Further, the scores of HAMD are associated with serum lipids. We mainly found that lipid profiles (especially the level of TC) can be served as a potential biomarker for patients with MDD to predict recent suicide attempts (within 2 weeks) and HAMD might be a concomitant indicator of the intensity of suicidal attempts.

Our findings are consistent with other studies. For instance, Maes et al. demonstrated an association between patients diagnosed with MDD and changes in lipid metabolism, such as decreased serum HDL-c levels and increased TG levels $(14,39)$. Furthermore, a study investigating adults aged between 45 and 65 revealed that ascending levels of TG were at risk for suicidal ideation (23). Hegerl et al. documented that the serum LDLc level of MDD patients was positively correlated with selfkilling (40). The mechanism of the association between lipid profiles in MDD patients and suicide attempts remains unclear and understudied. A review of metabolic syndrome and mood disorders showed an attempt made to link mood disorders with lowered HDL-c level and increased TG level in the organism (41).

Age comparisons demonstrate a significant and confounding effect on blood lipids, and age can be shown to relate positively to concentration levels of lipid profiles (35). We divided the patients into three age cohorts. After controlling for age, gender and BMI, it was found that only TC levels in MDD patients of

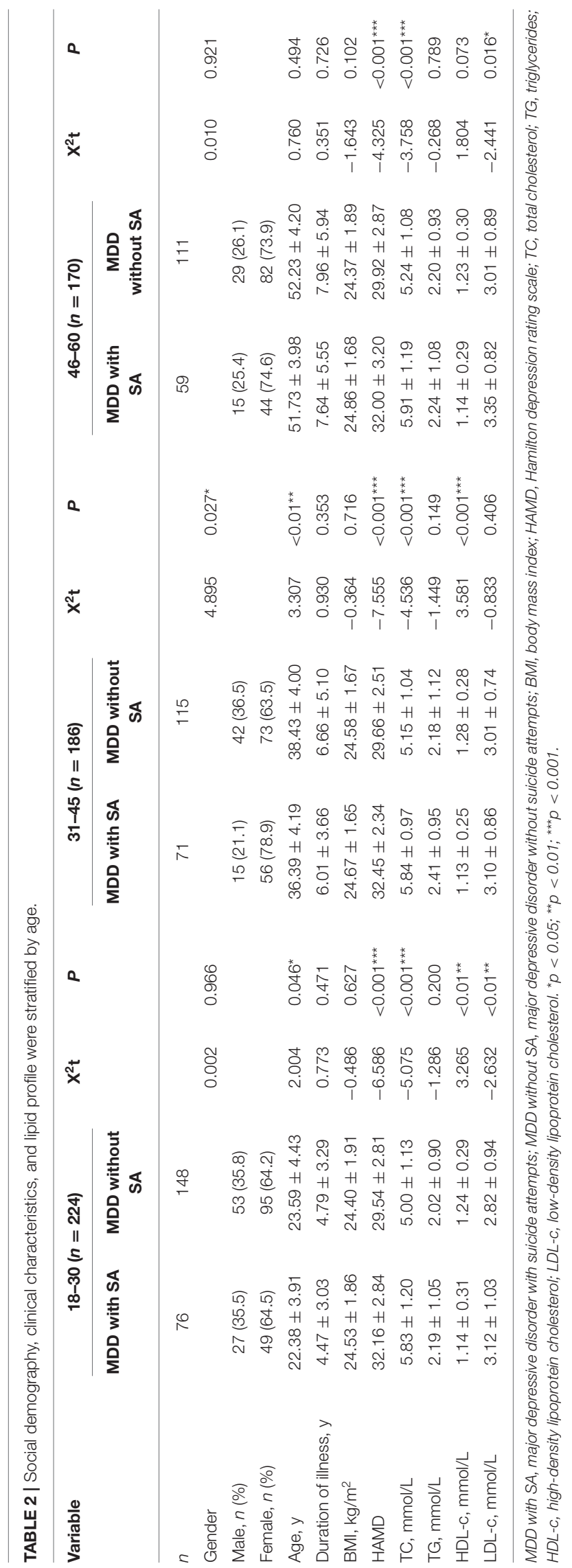


TABLE 3 | Association between lipid profile and suicide attempt: multiple logistic regression analyses.

\begin{tabular}{|c|c|c|c|c|c|c|c|c|}
\hline & \multicolumn{2}{|c|}{ Total $(n=580)^{\mathrm{a}}$} & \multicolumn{2}{|c|}{$18-30(n=224)^{b}$} & \multicolumn{2}{|c|}{$31-45(n=186)^{c}$} & \multicolumn{2}{|c|}{$46-60(n=170)^{d}$} \\
\hline & OR & $P$ & OR & $P$ & OR & $P$ & OR & $\boldsymbol{P}$ \\
\hline TC & 1.897 & $<0.001^{\star \star \star}$ & 1.961 & $<0.001^{\star \star \star}$ & 2.344 & $<0.001^{\star \star \star}$ & 1.654 & $<0.01^{\star *}$ \\
\hline TG & 0.907 & 0.325 & 0.831 & 0.294 & 0.977 & 0.891 & 0.879 & 0.486 \\
\hline HDL-C & 0.361 & $<0.01^{\star *}$ & 0.297 & $0.024^{*}$ & 1.633 & $0.012^{\star}$ & 0.716 & 0.581 \\
\hline LDL-C & 0.819 & 0.123 & 0.828 & 0.350 & 0.536 & $0.023^{*}$ & 1.088 & 0.716 \\
\hline
\end{tabular}

Stratified analysis across diagnostic groups based on continuous lipid profile variables. Each row represents a separate model adjusted for age, gender, and BMI (body mass index). OR, odds ratio; TC, total cholesterol; TG, triglycerides; HDL-c, high-density lipoprotein cholesterol; LDL-c, low-density lipoprotein cholesterol.

Number of suicide attempters, non-attempters: ${ }^{a} 206,374 ;{ }^{b} 76,148 ;{ }^{c} 71,115 ;{ }^{d} 59,111 .{ }^{*} p<0.05 ;{ }^{* *} p<0.01 ;{ }^{* * *} p<0.001$.

TABLE 4 | Inter-correlations between HAM-D and lipid profiles.

\begin{tabular}{lccccc}
\hline Variables & & TC & TG & HDL-c & LDL-c \\
\hline HAMD & $r$ & $\begin{array}{c}0.584 \\
<0.001^{*}\end{array}$ & $\begin{array}{c}0.209 \\
<0.001^{*}\end{array}$ & $\begin{array}{c}-0.200 \\
<0.001^{*}\end{array}$ & $\begin{array}{c}0.386 \\
<0.001^{*}\end{array}$ \\
\hline
\end{tabular}

HAMD, Hamilton depression rating scale; TC, total cholesterol; TG, triglycerides; $H D L-c$, high-density lipoprotein cholesterol; LDL-C, low-density lipoprotein cholesterol. ${ }^{\star}$ Bonferroni corrected $p<0.05 / 4=0.0125$.

different age groups maintained a significant relationship with suicide attempts $(p<0.01)$; TC levels could, therefore, be used as an indicator to predict the suicide risk of patients diagnosed with first-episode MDD.

A multitude of studies have shown that in subjects with suicidal thoughts, a decrease in TC concentration was noted, and there is a strong link between low TC level and suicide attempts $(25,42,43)$. Notwithstanding, the results from the current research suggest the opposite. This study has found that ascending TC concentrations in lipid profiles are positively correlated with the severity of suicidal tendencies in individuals suffering from first-episode MDD aged from 18 to 60 . It is noteworthy that in the further logic analysis, TC levels showed statistical significance in three age-stratified, firstepisode MDD patients (all $p<0.01$ ), indicating the feasibility and relative reliability of using TC levels as an independent predictor of suicide risk in MDD patients. Cross-sectional research concluded that TC and TG levels were not reduced in those who had attempted suicide and that, conversely, patients with excessive TC levels were at risk of suicidal behavior (21). Similarly, a study has indicated that higher cholesterol levels are reportedly linked to the risk of increased suicidal behavior in depressed individuals (30). Cholesterol in human serum is involved in the production of myelin, transmembrane exchange, synthesis of steroid hormones, and expression of neurotransmitter receptors, thus, yielding disease and behavior through its vital role in various aspects (44). Additionally, Asellus et al. demonstrated that that increased levels of 5hydroxyindoleacetic acid (5-HIAA), a well-known component of suicide risk related factor, in the cerebrospinal fluid had a tendency to be linked to higher TC level of lipid (45, 46). We propose that increased levels of TC constitute a risk factor for increased suicidal tendencies in first-episode MDD patients.

Several reasons account for the discrepancy between our findings and previous studies. First, all MDD patients we selected were first-episode non-medication patients. Although the results of studies on blood lipids and suicidal behaviors in MDD patients appear to different, the majority of research only unveiled the relationship between blood lipids and suicide attempts in MDD patients. A range of previous research, revealing the fact that concentration of serum lipid and suicidality in MDD patients are related to the use of anti-depressant (31), obviously tend to ignore the underlying interplay of anti-depressant drugs on the measurement of lipid profiles. For example, Shahsavand Ananloo et al. found that the level of TC decreased in patients with major depression by using fluoxetine (47). Therefore, the exclusion of subjects with anti-depressant use in our research would minimize the implication of medication. Second, the suicide attempts of the patients were all those who had attempted suicide within 2 weeks, and the timing of suicidality was shorter. Previous studies were heterogeneous in the definitions of result (suicidal ideation, attempt, propensity), and associated (recent or lifetime) timelines $(48,49)$. For this, we conducted on patients who had recently attempted suicide (within 2 weeks). Furthermore, the increased level of TC can lead to inflammation and some inflammatory factors can reach the central nervous system (CNS), which may take part in the development of psychiatric diseases and lead to some symptoms via acting on the neural structure and function (50). Studies have shown that patients with suicide ideation and behavior expressed changed inflammation in blood and cerebrospinal fluid (51-53). Although the causality between inflammation and suicide behavior is not known, it may suggest the underlying mechanism.

Correlation analysis suggested that HAMD is related to the lipid profile. Thus, we thought HAMD might be a concomitant indicator of the intensity of suicide attempt in first-episode MDD patients. The scores of HAMD reflect the degree of depression, and with the development of depression, the occurrence of suicidal attempts increased. Emotion processing difficulties can also lead to suicide attempts. Alexithymia is a personality trait that is characterized by having difficulty in identifying and expressing emotions and in using cognitive methods that address external events rather than internal experiences. Alexithymia is considered to be a susceptible factor affecting the onset and 
course of many mental disorders, which is common in subjects with psychiatric disorders (54), such as MDD. Interestingly, De Berardis et al. suggested that alexithymic individuals exhibited altered serum lipid levels, which is associated with suicidal ideation $(55,56)$. Alexithymic can increase the risk of developing depressive symptoms. Thus, we speculated that with the development of depressive symptoms, a difficulty when processing emotions might occur, such as alexithymia, which leads to the change of the serum TC levels and in turn an attempt at suicide.

Our study presents some limitations. First of all, this study did not differentiate the subtypes of MDD (i.e., with melancholic or mixed or anxious features or seasonal MDD). Second, this study is not able to deduce the causal relationship due to the design of cross-sectional. For the sake of more profound exploration of the causal relationship between these variables in patients with MDD, further studies should focus on longitudinal study design. Future studies should involve more patients with MDD at different stages. Third, confounding factors crucial to the study were not gathered, incorporating smoking, alcohol abuse, eating habits, nutritional status, and physical activity. Finally, our research results should be considered preliminary because of the deficiency of the healthy control group. Our results are expected to be confirmed, replicated, and extended in future studies.

\section{CONCLUSION}

Compared with non-suicidal MDD patients, patients with suicide attempts are more vulnerable to having higher HAMD scores, higher TC, TG, and LDL-c levels, lower HDL-c level, suggesting that lipid profiles may be a promising biomarkers for firstepisode MDD suicide risk. Furthermore, after controlling for other variables and age stratification, the OR of TC was still significant, and for first-episode MDD patients of all ages, the TC level in the body is an indicator with the importance of whether or not patients have recently attempted suicide. The results of this study indicate that routinely screening blood lipid levels in people during their first episode of MDD could be a way of evaluating the risk of suicide. Moreover, this represents a new and potentially valuable field for future medical professionals in assessing suicide

\section{REFERENCES}

1. Battle DE. Diagnostic and statistical manual of mental disorders (DSM). Codas. (2013) 25:191-2. doi: 10.1590/s2317-17822013000200017

2. Dong M, Wang SB, Li Y, Xu DD, Ungvari GS, Ng CH, et al. Prevalence of suicidal behaviors in patients with major depressive disorder in China: a comprehensive meta-analysis. J Affect Disord. (2018) 225:32-9. doi: 10.1016/j.jad.2017.07.043

3. Friedrich MJ. Depression is the leading cause of disability around the world. JAMA. (2017) 317:1517. doi: 10.1001/jama.2017.3826

4. Arensman E, Scott V, De Leo D, Pirkis J. Suicide and suicide prevention from a global perspective. Crisis. (2020) 41(Suppl. 1):S3-7. doi: 10.1027/0227-5910/a000664

5. Kim YK, Myint AM. Clinical application of low serum cholesterol as an indicator for suicide risk in major depression. J Affect Disord. (2004) 81:161-6. doi: 10.1016/S0165-0327(03)00166-6 risk in MDD patients using serum lipid profiles. Given the lack of research on the relationship between blood lipid levels and suicide in MDD patients, the mechanism is unclear, and more research is needed to elucidate the underlying relationships.

\section{DATA AVAILABILITY STATEMENT}

The raw data supporting the conclusions of this article will be made available by the authors, without undue reservation.

\section{ETHICS STATEMENT}

The studies involving human participants were reviewed and approved by the Affiliated Kangning Hospital of Wenzhou Medical University research ethics committee. The patients/participants provided their written informed consent to participate in this study.

\section{AUTHOR CONTRIBUTIONS}

SYZ and KZ: put forward the presented idea and writing the original draft. XS: designed the research protocol. HKS, SYD, and XMM: data curation and formal analysis. JJC, FY, MZX, WR, and JYL: revised the paper and help do some work. XYZ: gave guidance. All authors discussed the results and contributed to the final manuscript.

\section{FUNDING}

This work was supported by the Wenzhou Science and Technology Project (Y20180115), the Natural Science Foundation of Zhejiang Province (LQ18H090009), the medical and health research project of Zhejiang province (2020KY926), and Zhejiang Province Science and Technology Plan Research and Xinmiao Talent Program (2020R413073).

\section{ACKNOWLEDGMENTS}

The authors would like to thank all the patients who participated and the study funders.

6. De Berardis D, Fornaro M, Orsolini L, Ventriglio A, Vellante F, Di Giannantonio M. Emotional dysregulation in adolescents: implications for the development of severe psychiatric disorders, substance abuse, and suicidal ideation and behaviors. Brain Sci. (2020) 10:591. doi: 10.3390/brainsci10090591

7. De Berardis D, Fornaro M, Valchera A, Cavuto M, Perna G, Di Nicola M, et al. Eradicating suicide at its roots: preclinical bases and clinical evidence of the efficacy of ketamine in the treatment of suicidal behaviors. Int J Mol Sci. (2018) 19:28888. doi: 10.3390/ijms19102888

8. Orsolini L, Latini R, Pompili M, Serafini G, Volpe U, Vellante F, et al. Understanding the complex of suicide in depression: from research to clinics. Psychiatry Investig. (2020) 17:207-21. doi: 10.30773/pi.2019.0171

9. Wagner CJ, Musenbichler C, Bohm L, Farber K, Fischer AI, von Nippold F, et al. LDL cholesterol relates to depression, its severity, and the prospective course. Prog Neuropsychopharmacol Biol Psychiatry. (2019) 92:405-11. doi: 10.1016/j.pnpbp.2019.01.010 
10. Pandey GN. Biological basis of suicide and suicidal behavior. Bipolar Disord. (2013) 15:524-41. doi: 10.1111/bdi.12089

11. da Graça Cantarelli M, Nardin P, Buffon A, Eidt MC, Antônio Godoy L, Fernandes BS, et al. Serum triglycerides, but not cholesterol or leptin, are decreased in suicide attempters with mood disorders. J Affect Disord. (2015) 172:403-9. doi: 10.1016/j.jad.2014.10.033

12. Baek JH, Kang ES, Fava M, Mischoulon D, Nierenberg AA, Yu BH, et al. Serum lipids, recent suicide attempt and recent suicide status in patients with major depressive disorder. Prog Neuropsychopharmacol Biol Psychiatry. (2014) 51:113-8. doi: 10.1016/j.pnpbp.2014.01.018

13. Brown BG, Zhao X-Q, Cheung MC. Should both HDL-C and LDL-C be targets for lipid therapy? A review of current evidence. J Clin Lipidol. (2007) 1:88-94. doi: 10.1016/j.jacl.2007.02.004

14. Maes M, Smith R, Christophe A, Vandoolaeghe E, Van Gastel A, Neels H, et al. Lower serum high-density lipoprotein cholesterol (HDL-C) in major depression and in depressed men with serious suicidal attempts: relationship with immune-inflammatory markers. Acta Psychiatr Scand. (1997) 95:212-21. doi: 10.1111/j.1600-0447.1997.tb09622.x

15. Chen CC, Lu FH, Wu JS, Chang CJ. Correlation between serum lipid concentrations and psychological distress. Psychiatry Res. (2001) 102:153-62. doi: 10.1016/S0165-1781(01)00231-1

16. Maes M, Delanghe J, Meltzer HY, Scharpe S, D’Hondt P, Cosyns P. Lower degree of esterification of serum cholesterol in depression: relevance for depression and suicide research. Acta Psychiatr Scand. (1994) 90:252-8. doi: 10.1111/j.1600-0447.1994.tb01589.x

17. Mead GE, Morley W, Campbell P, Greig CA, McMurdo M, Lawlor DA. Exercise for depression. Cochrane Database Syst Rev. (2009). CD004366. doi: 10.1002/14651858.CD004366.pub4

18. Neaton JD, Blackburn H, Jacobs D, Kuller L, Lee DJ, Sherwin R, et al. Serum cholesterol level and mortality findings for men screened in the multiple risk factor intervention trial. Multiple risk factor intervention trial research group. Arch Intern Med. (1992) 152:1490-500. doi: 10.1001/archinte.1992.00400190110021

19. Lindberg G, Råstam L, Gullberg B, Eklund GA. Low serum cholesterol concentration and short term mortality from injuries in men and women. BMJ. (1992) 305:277-9. doi: 10.1136/bmj.305.6848.277

20. de Leon J, Mallory P, Maw L, Susce MT, Perez-Rodriguez MM, Baca-Garcia E. Lack of replication of the association of low serum cholesterol and attempted suicide in another country raises more questions. Ann Clin Psychiatry. (2011) 23:163-70.

21. Brunner J, Bronisch T, Pfister H, Jacobi F, Hofler M, Wittchen HU. High cholesterol, triglycerides, and body-mass index in suicide attempters. Arch Suicide Res. (2006) 10:1-9. doi: 10.1080/13811110500318083

22. Pompili M, Innamorati M, Lester D, Girardi P, Tatarelli R. Nearly lethal resuscitated suicide attempters have no low serum levels of cholesterol and triglycerides. Psychol Rep. (2010) 106:785-90. doi: 10.2466/pr0.106.3.785-790

23. Oh J, Kim TS. Serum lipid levels in depression and suicidality: the Korea national health and nutrition examination survey (KNHANES) 2014. J Affect Disord. (2017) 213:51-8. doi: 10.1016/j.jad.2017.02.002

24. Muldoon MF, Manuck SB, Matthews KA. Lowering cholesterol concentrations and mortality: a quantitative review of primary prevention trials. BMJ. (1990) 301:309-14. doi: 10.1136/bmj.301.6755.815

25. Golier JA, Marzuk PM, Leon AC, Weiner C, Tardiff K. Low serum cholesterol level and attempted suicide. Am J Psychiatry. (1995) 152:419-23. doi: 10.1176/ajp.152.3.419

26. Apter A, Laufer N, Bar-Sever M, Har-Even D, Ofek H, Weizman A. Serum cholesterol, suicidal tendencies, impulsivity, aggression, and depression in adolescent psychiatric inpatients. Biol Psychiatry. (1999) 46:532-41. doi: 10.1016/S0006-3223(98)00345-X

27. D'Ambrosio V, Salvi V, Bogetto F, Maina G. Serum lipids, metabolic syndrome and lifetime suicide attempts in patients with bipolar disorder. Prog Neuropsychopharmacol Biol Psychiatry. (2012) 37:136-40. doi: 10.1016/j.pnpbp.2011.12.009

28. Huang TL. Serum lipid profiles in major depression with clinical subtypes, suicide attempts and episodes. J Affect Disord. (2005) 86:75-9. doi: $10.1016 /$ j.jad.2004.11.005
29. Huang TL. Serum cholesterol levels in mood disorders associated with physical violence or suicide attempts in Taiwanese. Chang Gung Med J. (2001) 24:563-8.

30. Fiedorowicz JG, Coryell WH. Cholesterol and suicide attempts: a prospective study of depressed inpatients. Psychiatry Res. (2007) 152:11-20. doi: 10.1016/j.psychres.2006.09.003

31. Kuwano N, Kato TA, Setoyama D, Sato-Kasai M, Shimokawa N, Hayakawa $\mathrm{K}$, et al. Tryptophan-kynurenine and lipid related metabolites as blood biomarkers for first-episode drug-naïve patients with major depressive disorder: an exploratory pilot case-control study. J Affect Disord. (2018) 231:74-82. doi: 10.1016/j.jad.2018.01.014

32. Boston PF, Dursun SM, Reveley MA. Cholesterol and mental disorder. $\mathrm{Br} J$ Psychiatry. (1996) 169:682-9. doi: 10.1192/bjp.169.6.682

33. Sullivan PF, Joyce PR, Bulik CM, Mulder RT, Oakley-Browne M. Total cholesterol and suicidality in depression. Biol Psychiatry. (1994) 36:472-7. doi: 10.1016/0006-3223(94)90643-2

34. Shen Y, Wu F, Zhou Y, Ma Y, Huang X, Ning Y, et al. Association of thyroid dysfunction with suicide attempts in first-episode and drug naïve patients with major depressive disorder. J Affect Disord. (2019) 259:180-5. doi: 10.1016/j.jad.2019.08.067

35. Bocchetta A, Chillotti C, Carboni G, Oi A, Ponti M, Del Zompo M. Association of personal and familial suicide risk with low serum cholesterol concentration in male lithium patients. Acta Psychiatr Scand. (2001) 104:3741. doi: 10.1034/j.1600-0447.2001.00374.x

36. Hamilton M. A rating scale for depression. J Neurol Neurosurg Psychiatry. (1960) 23:56-62. doi: 10.1136/jnnp.23.1.56

37. Hamilton M. The assessment of anxiety states by rating. Br J Med Psychol. (1959) 32:50-5. doi: 10.1111/j.2044-8341.1959.tb00467.x

38. Joiner TE, Jr., Steer RA, Brown G, Beck AT, Pettit JW, et al. Worstpoint suicidal plans: a dimension of suicidality predictive of past suicide attempts and eventual death by suicide. Behav Res Ther. (2003) 41:1469-80. doi: 10.1016/S0005-7967(03)00070-6

39. Maes M, Delanghe J, Scharpe S, Meltzer HY, Cosyns P, Suy E, et al. Haptoglobin phenotypes and gene frequencies in unipolar major depression. Am J Psychiatry. (1994) 151:112-6. doi: 10.1176/ajp.151.1.112

40. Hegerl U, Gallinat J, Juckel G. Event-related potentials. Do they reflect central serotonergic neurotransmission and do they predict clinical response to serotonin agonists? J Affect Disord. (2001) 62:93-100. doi: 10.1016/S0165-0327(00)00353-0

41. de Melo LGP, Nunes SOV, Anderson G, Vargas HO, Barbosa DS, Galecki P, et al. Shared metabolic and immune-inflammatory, oxidative and nitrosative stress pathways in the metabolic syndrome and mood disorders. Prog Neuropsychopharmacol Biol Psychiatry. (2017) 78:34-50. doi: 10.1016/j.pnpbp.2017.04.027

42. Segoviano-Mendoza M, Cárdenas-de la Cruz M, Salas-Pacheco J, VázquezAlaniz F, La Llave-León O, Castellanos-Juárez F, et al. Hypocholesterolemia is an independent risk factor for depression disorder and suicide attempt in Northern Mexican population. BMC Psychiatry. (2018) 18:7-. doi: $10.1186 / \mathrm{s} 12888-018-1596-\mathrm{z}$

43. Lalovic A, Levy E, Luheshi G, Canetti L, Grenier E, Sequeira A, et al. Cholesterol content in brains of suicide completers. Int $J$ Neuropsychopharmacol. (2007) 10:159-66. doi: 10.1017/S1461145706006663

44. Golomb BA, Criqui MH, White HL, Dimsdale JE. The UCSD statin study: a randomized controlled trial assessing the impact of statins on selected noncardiac outcomes. Control Clin Trials. (2004) 25:178-202. doi: 10.1016/j.cct.2003.08.014

45. Mann JJ, Malone KM, Sweeney JA, Brown RP, Linnoila M, Stanley B, et al. Attempted suicide characteristics and cerebrospinal fluid amine metabolites in depressed inpatients. Neuropsychopharmacology. (1996) 15:576-86. doi: 10.1016/S0893-133X(96)00102-9

46. Asellus P, Nordstrom P, Jokinen J. Cholesterol and CSF 5-HIAA in attempted suicide. J Affect Disord. (2010) 125:388-92. doi: 10.1016/j.jad.2010.02.111

47. Shahsavand Ananloo E, Ghaeli P, Kamkar MZ, Sadeghi M. Comparing the effects of fluoxetine and imipramine on total cholesterol, triglyceride, and weight in patients with major depression. Daru. (2013) 21:4. doi: $10.1186 / 2008-2231-21-4$ 
48. Wu S, Ding Y, Wu F, Xie G, Hou J, Mao P. Serum lipid levels and suicidality: a meta-analysis of 65 epidemiological studies. J Psychiatry Neurosci. (2016) 41:56-69. doi: 10.1503/jpn.150079

49. Park S, Yi KK, Na R, Lim A, Hong JP. No association between serum cholesterol and death by suicide in patients with schizophrenia, bipolar affective disorder, or major depressive disorder. Behav Brain Funct. (2013) 9:45. doi: 10.1186/1744-9081-9-45

50. Udina M, Castellví P, Moreno-España J, Navinés R, Valdés M, Forns $\mathrm{X}$, et al. Interferon-induced depression in chronic hepatitis C: a systematic review and meta-analysis. J Clin Psychiatry. (2012) 73:1128-38. doi: 10.4088/JCP.12r07694

51. Black C, Miller BJ. Meta-analysis of cytokines and chemokines in suicidality: distinguishing suicidal versus nonsuicidal patients. Biol Psychiatry. (2015) 78:28-37. doi: 10.1016/j.biopsych.2014.10.014

52. Lindqvist D, Janelidze S, Hagell P, Erhardt S, Samuelsson M, Minthon $\mathrm{L}$, et al. Interleukin- 6 is elevated in the cerebrospinal fluid of suicide attempters and related to symptom severity. Biol Psychiatry. (2009) 66:287-92. doi: 10.1016/j.biopsych.2009.01.030

53. O’Donovan A, Rush G, Hoatam G, Hughes BM, McCrohan A, Kelleher C, et al. Suicidal ideation is associated with elevated inflammation in patients with major depressive disorder. Depress Anxiety. (2013) 30:307-14. doi: 10.1002/da.22087
54. Leweke F, Leichsenring F, Kruse J, Hermes S. Is alexithymia associated with specific mental disorders? Psychopathology. (2012) 45:22-8. doi: 10.1159/000325170

55. De Berardis D, Fornaro M, Orsolini L, Valchera A, Carano A, Vellante F, et al. Alexithymia and suicide risk in psychiatric disorders: a mini-review. Front Psychiatry. (2017) 8:148. doi: 10.3389/fpsyt.2017.00148

56. De Berardis D, Serroni N, Marini S, Rapini G, Carano A, Valchera A, et al. Alexithymia, suicidal ideation, and serum lipid levels among drug-naïve outpatients with obsessive-compulsive disorder. Braz J Psychiatry. (2014) 36:125-30. doi: 10.1590/1516-4446-2013-1189

Conflict of Interest: The authors declare that the research was conducted in the absence of any commercial or financial relationships that could be construed as a potential conflict of interest.

Copyright (c) 2021 Zhou, Zhao, Shi, Sun, Du, Miao, Chen, Yang, Xing, Ran, Lao, Zhang, Wang and Tang. This is an open-access article distributed under the terms of the Creative Commons Attribution License (CC BY). The use, distribution or reproduction in other forums is permitted, provided the original author(s) and the copyright owner(s) are credited and that the original publication in this journal is cited, in accordance with accepted academic practice. No use, distribution or reproduction is permitted which does not comply with these terms. 